\title{
Optics of a two-stage collimation system
}

\author{
J. B. Jeanneret \\ CERN, CH-1211 Geneva, Switzerland \\ (Received 13 October 1998; published 21 December 1998)
}

\begin{abstract}
We derive the exact specification that a two-stage betatronic collimation insertion must satisfy to cut the halo of a proton beam down to its ultimate limit which is the aperture of the secondary collimators. Our result is a set of correlated phase advances between primary and secondary collimators. We then determine the number of jaws needed to reach a given level of performance. We also specify the optic of a momentum collimation insertion. [S1098-4402(98)00024-X]
\end{abstract}

PACS numbers: 29.27.Eg, 41.85.Si

\section{INTRODUCTION}

In superconducting proton colliders of both high energy and high beam current, the control of beam losses is mandatory. Local power deposition associated to beam losses can be larger than the quench level of superconducting magnets by several orders of magnitude [1]. In addition, the large size of the rings at high energy implies keeping the transverse size of the magnets small for obvious cost reasons. Therefore the geometrical aperture delimited by the vacuum chamber must be kept to its bare minimum. Beam losses are mostly related to beam dynamics. Not far above the dynamic aperture, the transverse motion of the particles becomes chaotic and can form a halo diffusing towards the geometrical aperture. The transverse extension of the halo is limited by absorbing these protons in collimators that are made of metallic blocks, which are called jaws. The jaws inserted close to the beam are called primary collimators and define the primary aperture which is normally chosen to be larger or equal to the dynamic aperture in order not to intercept stable particles. At all energies the absorption of protons in primary jaws is substantially distant from unity [1]. Protons which are not absorbed can be scattered elastically off the jaw, thus forming a secondary halo which can also induce quenches. Secondary jaws are therefore necessary to limit the extension of the secondary halo to a value smaller than the geometrical aperture or, otherwise said, to allow for the choice of a small but safe geometrical aperture.

The geometrical size of the secondary halo, normalized to the aperture of the collimators, depends on the number of jaws, on their relative locations, and on the optic of the insertion where they are installed.

An exact treatment of a two-stage collimation system considered as an optical device, i.e., disregarding true scattering in collimator jaws, exists for the onedimensional (1D) case and in the special two-dimensional (2D) case of an optic with equal phase advance in the two transverse dimensions [2]. The problem of a 2D system with an arbitrary optic was solved with numerical methods in conjunction with the approximate concept of phase modulation with some success $[2,3]$, but without cutting the amplitude of the secondary halo down to the ultimate limit of the aperture of the secondary collimators. Existing collimation systems in high energy proton machines are, or were, all made of two 1D systems (see the caption of Fig. 3). No calculated or measured performance exists. The sole documented case is the HERA collimation system [4]. In all these studies, taking apart the 1D case, the best arrangement of jaws was found for a predefined optic. The solutions found are therefore some kind of local minima, in the hypothetical space of all possible optics.

In this paper, we do not consider a particular optic. Rather, we derive the optical constraints between a primary jaw and its associated secondary jaws which minimize the size of the secondary halo issued from the primary jaw. The constraints are expressed by correlated betatronic phase advances between primary and secondary jaws. The end result is an exact specification that an optic must satisfy to provide an optimum collimation system for a given number of jaws.

\section{DEFINITION AND NOTATIONS}

We use horizontal and vertical betatron coordinates as well as horizontal dispersion normalized with the transformations

$$
\vec{X}=N_{x} \vec{x}, \quad \vec{Y}=N_{y} \vec{y}, \quad \text { and } \quad \vec{\chi}=N_{x} \vec{D},
$$

which expand as

$$
\left(\begin{array}{c}
X \\
X^{\prime}
\end{array}\right)=\frac{1}{\sigma_{x}}\left(\begin{array}{cc}
1 & 0 \\
\alpha_{x} & \beta_{x}
\end{array}\right)\left(\begin{array}{c}
x \\
x^{\prime}
\end{array}\right),
$$

for $X$ coordinates, and similarly for $Y$. We group $\vec{X}$ and $\vec{Y}$ in four-vectors noted $\vec{A}=\left(X, X^{\prime}, Y, Y^{\prime}\right)$. The vector $\vec{A}$ is transported between two locations with $\vec{A}_{2}=M_{12} \vec{A}_{1}$. The transfer matrix $M_{12}$ is made of two clockwise rotations, one for each proper plane, where the angles of rotation $\mu_{x}$ and $\mu_{y}$ are the betatronic phase advances; i.e.,

$$
M_{12}=\left(\begin{array}{cccc}
\cos \mu_{x} & \sin \mu_{x} & 0 & 0 \\
-\sin \mu_{x} & \cos \mu_{x} & 0 & 0 \\
0 & 0 & \cos \mu_{y} & \sin \mu_{y} \\
0 & 0 & -\sin \mu_{y} & \cos \mu_{y}
\end{array}\right) \text {. }
$$


The normalized invariant amplitudes are

$$
A_{x}=\left(X^{2}+X^{\prime 2}\right)^{1 / 2}, \quad A_{y}=\left(Y^{2}+Y^{\prime 2}\right)^{1 / 2},
$$

which can be added to form the combined betatronic invariant amplitude

$$
A=\left(A_{x}^{2}+A_{y}^{2}\right)^{1 / 2}
$$

\section{BETATRON COLLIMATION}

We first consider circular collimators in normalized coordinates. The normalized apertures of the primary and secondary collimators are $n_{1}$ and $n_{2}$ rms transverse beam units, respectively. These numbers are fixed in our problem in the sense that $n_{1}$ cannot be varied to optimize a collimation system but must rather fit to external parameters like the dynamic aperture or the effective geometrical aperture of the ring. Similarly, we will see in Sec. III A that the relative retraction $\left(n_{2}-\right.$ $\left.n_{1}\right) / n_{1}$ must be kept constant once it has been chosen and shall therefore be substantially larger than, for example, local closed orbit changes. We use the approximation of slow transverse diffusion of the primary halo, with the consequence that the impact parameter at the primary collimator is small compared to $n_{1}$. More precisely, we consider the impact points to be at the surface of the collimator while both betatronic oscillations are at their maxima; i.e., $X_{0}^{\prime}=Y_{0}^{\prime}=0$ with $X_{0}^{2}+Y_{0}^{2}=n_{1}^{2}$. In Sec. III A, we will minimize the extension of the secondary halo after it is cut by the secondary collimators treated as black absorbers, thus neglecting the formation of a tertiary halo.

Using the transverse azimuth $\alpha$ to define the point of impact on the primary collimator, the coordinates of the particles before scattering are

$$
\vec{A}_{0}=\left(n_{1} \cos \alpha, 0, n_{1} \sin \alpha, 0\right) .
$$

The scattering process adds an arbitrary value to $X_{0}^{\prime}$ and $Y_{0}^{\prime}$, using here, for simplicity, an isotropic distribution. With the use of the polar coordinates $K$ and $\phi$ in the $X_{0}^{\prime}-Y_{0}^{\prime}$ plane (see Fig. 1), the coordinates at the primary collimator after scattering are

$$
\vec{A}_{1}=\left(n_{1} \cos \alpha, K \cos \phi, n_{1} \sin \alpha, K \sin \phi\right) .
$$

\section{A. Phase advances}

For arbitrary $\alpha$ and $\phi$ angles, we transport the particle with $\vec{A}_{2}=M_{12} \vec{A}_{1}$ using Eqs. (3) and (7) to a location of yet unspecified phase advances $\mu_{x}$ and $\mu_{y}$ where a secondary collimator is located and get

$$
\vec{A}_{2}=\left(\begin{array}{c}
n_{1} \cos \alpha \cos \mu_{x}+K \cos \phi \sin \mu_{x} \\
-n_{1} \cos \alpha \sin \mu_{x}+K \cos \phi \cos \mu_{x} \\
n_{1} \sin \alpha \cos \mu_{y}+K \sin \phi \sin \mu_{y} \\
-n_{1} \sin \alpha \sin \mu_{y}+K \sin \phi \cos \mu_{y}
\end{array}\right) .
$$
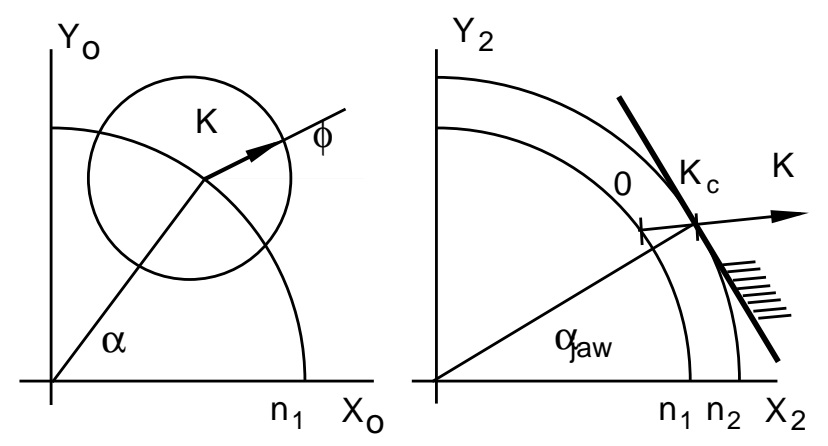

FIG. 1. The line of the scattered particles at the primary collimator parametrized with $\left(n_{1}, \alpha, K, \phi\right)$ transforms at the location of a secondary collimator to another line which crosses the circle of radius $n_{2}$ when $K=K_{c}$ whatever $\alpha$ and $\phi$; see Eq. (10). A flat jaw at azimuth $\alpha_{\text {jaw }}$ is sufficient to cut at amplitude $A=n_{2}$; see text.

The phases $\mu_{x}$ and $\mu_{y}$ are the free parameters of our problem. The efficiency of the secondary collimator is measured by the smallest amplitude $A_{2 \text {,cut }}$ that it can intercept. $A_{2 \text {,cut }}$ is minimized if $X_{2}$ and $Y_{2}$ are maximized, because the aperture of the secondary collimators is fixed to $n_{2}$. Using the invariance of $A_{x, 2}$ and $A_{y, 2}$, this condition is equivalent to asking for $X_{2}^{\prime}=Y_{2}^{\prime}=0$. With these two conditions in Eq. (8) we get

$$
\tan \mu_{x}=\frac{K \cos \phi}{n_{1} \cos \alpha}, \quad \tan \mu_{y}=\frac{K \sin \phi}{n_{1} \sin \alpha} .
$$

These conditions allow one to compute the sole free parameters $\mu_{x}=\mu_{x}(\alpha, \phi, K)$ and $\mu_{y}=\mu_{y}(\alpha, \phi, K)$. While $\alpha$ and $\phi$ are free variables, $K$ is restricted to its maximum allowed value corresponding to the smallest possible $A_{2, \text { cut }}=n_{2}$ (see Fig. 1). This is obtained by the substitution of Eqs. (9) into Eq. (8) with again $X_{2}^{\prime}=$ $Y_{2}^{\prime}=0$. We get

$$
K=K_{c}=\sqrt{n_{2}^{2}-n_{1}^{2}},
$$

which is independent of both $\alpha$ and $\phi$. Writing

$$
\begin{aligned}
\tan \mu_{0} & =K_{c} / n_{1}=\left(n_{2}^{2}-n_{1}^{2}\right)^{1 / 2} / n_{1} \\
\text { or } \quad \cos \mu_{0} & =n_{1} / n_{2},
\end{aligned}
$$

Eqs. (9) become

$$
\tan \mu_{x}=\tan \mu_{0} \frac{\cos \phi}{\cos \alpha}, \quad \tan \mu_{y}=\tan \mu_{0} \frac{\sin \phi}{\sin \alpha} .
$$

The normalized angle $K_{c}$ is the largest scattering angle which passes the secondary jaw and, as it shall be, the corresponding largest amplitude is $A_{1, \max }=A_{2, \mathrm{cut}}=\left(n_{1}^{2}+\right.$ $\left.K_{c}^{2}\right)^{1 / 2}=n_{2}$. The two conditions stated by Eqs. (12) form our central result. They fix univocally the location of a secondary jaw to cut the secondary amplitudes to its lower limit $A_{2}=n_{2}$ for $\alpha$ and $\phi$. They are governed by the single parameter $\mu_{0}$ (see also Table III). The phase $\mu_{0}$ depends on the choice of the ratio $n_{1} / n_{2}$. Therefore, 
this ratio must be fixed before choosing the optic of a cleaning insertion. Its value also fixes the location of all secondary collimators. These formulas indicate that an optimum collimation for all possible $\alpha$ and $\phi$ would need an infinity of collimators, with an optic able to offer an infinity of pairs of phase advances $\left(\mu_{x}, \mu_{y}\right)$ which satisfy Eqs. (12).

Before compromising on the number of collimators, it must be noticed that for given $(\alpha, \phi)$, the secondary collimator at $\left(\mu_{x}, \mu_{y}\right)$ need not be circular. A single flat jaw at the $X-Y$ azimuth $\alpha_{J}=\tan ^{-1}\left(Y_{2} / X_{2}\right)$ is sufficient (see Fig. 1). With Eq. (8), the azimuth of the jaw must be

$$
\tan \alpha_{J}=\frac{\sin \alpha \cos \mu_{y}+\tan \mu_{0} \sin \phi \sin \mu_{y}}{\cos \alpha \cos \mu_{x}+\tan \mu_{0} \cos \phi \sin \mu_{x}} .
$$

In practice, the transverse adjustment of the jaws, i.e., at either $n_{1}$ or $n_{2}$ beam units of the central orbit, can be made only by the use of an opposite jaw in the same tank, together forming a pair with their respective azimuths $\alpha_{J}$ and $\alpha_{J}+\pi$ (see [5]). Therefore, the determination of $\alpha_{J}$ by its tangent in Eq. (13) in the range $[-\pi / 2, \pi / 2]$ modulo $\pi$ is univocal. For later use in Sec. III B we compute also

$$
\cos \alpha_{J}=\frac{n_{1}}{n_{2}} \frac{\cos \alpha}{\cos \mu_{x}}, \quad \sin \alpha_{J}=\frac{n_{1}}{n_{2}} \frac{\sin \alpha}{\cos \mu_{y}} .
$$

The result is obtained for $\cos \alpha_{J}$ with $\cos \alpha_{J}=$ $X_{2} / n_{2}=\left(n_{1} / n_{2}\right) \cos \mu_{x}\left(\cos \alpha+K_{c} \tan \mu_{x} \cos \phi\right) \quad$ and Eq. (14) then by rewriting Eq. (12) as $K_{c} / n_{1}=$ $\tan \mu_{x} \cos \alpha / \cos \phi$ and using $1+\tan ^{2} \mu_{x}=1 / \cos ^{2} \mu_{x}$. The derivation is identical for $\sin \alpha_{J}=Y_{2} / n_{2}$.

From now on we will consider flat collimators only.

\section{B. Geometry of the secondary halo in the phase space}

For a given pair of primary impact and scattering angles $(\alpha, \phi)$ and its associated secondary jaw located at optimized phase advances $\left(\mu_{x}, \mu_{y}\right)$ oriented at the transverse azimuth $\alpha_{J}$ obtained, respectively, with Eqs. (12) and (13), we compute the domain of scattering angles at the primary collimator which are projected at the edge of the secondary jaw. The scattering angles in the $X_{1}^{\prime}-Y_{1}^{\prime}$ plane are parametrized with the free azimuth $\psi$

$$
K_{x}=K \cos \psi, \quad K_{y}=K \sin \psi .
$$

The edge of the secondary jaw is parametrized with

$$
X_{2} \cos \alpha_{J}+Y_{2} \sin \alpha_{J}=n_{2} .
$$

We rewrite $X_{2}$ and $Y_{2}$ from Eq. (8),

$$
\begin{aligned}
& X_{2}=n_{1} \cos \alpha \cos \mu_{x}+K_{x} \sin \mu_{x}, \\
& Y_{2}=n_{1} \sin \alpha \cos \mu_{y}+K_{y} \sin \mu_{y} .
\end{aligned}
$$

With Eqs. (17), (18), and (14) in Eq. (16) we get

$$
K_{x} n_{1} \cos \alpha \tan \mu_{x}+K_{y} n_{1} \sin \alpha \tan \mu_{y}=n_{2}^{2}-n_{1}^{2} .
$$

Using Eqs. (10), (12), and the definition (11), we finally get

$$
K_{x} \cos \phi+K_{y} \sin \phi=K_{c},
$$

which is the normalized equation of a line with $K_{c}$ the shortest distance to the origin and $\phi$ its slope. With this result the effect of an optimized secondary jaw is easily interpreted. With $K_{c}$ the smallest scattering angle cut when $\psi=\phi$ (remembering that the optimization was done for the scattering angle $\phi$ ), the line of Eq. (20) delimits a half-plane of scattering angles $K_{x} \cos \phi+$ $K_{y} \sin \phi>K_{c}$ intercepted by the jaw and the complementary half-plane $K_{x} \cos \phi+K_{y} \sin \phi<K_{c}$ passing the jaw. Several secondary jaws labeled with their corresponding central scattering azimuth $\left(\alpha, \phi_{i}, i \in\left[1, m_{s}\right]\right)$ define a polygon of order $m_{s}$ (if $m_{s} \geq 3$ ) which delimits the area of scattering angles which are not intercepted. The secondary halo is therefore delimited in the 4D phase space at the location of the primary jaw by a 2D polygon, labeled by the index $m_{s}$, located in a plane parallel to the axes $X^{\prime}$ and $Y^{\prime}$ at $\left(X_{1}=n_{1} \cos \alpha, Y_{1}=\right.$ $\left.n_{1} \sin \alpha\right)$. This polygon has an inscribed circle of radius $K_{c}$. The largest amplitude of the secondary halo is therefore $A_{\max }^{2}\left(m_{s}\right)=n_{1}^{2}+K_{\max }^{2}\left(m_{s}\right)$ with $K_{\max }\left(m_{s}\right)$ the distance of the most remote apex of the polygon relative to the origin $X^{\prime}=Y^{\prime}=0$ (see Fig. 2).

In addition to the optimization made by using the correlated phase advances of Eq. (12), a second optimization is now made by requesting that for given $m_{s}$ the polygon is made regular. This minimizes both $K_{\max }\left(m_{s}\right)$ and the surface of the polygon. The scattering angles $\phi_{i}$ used to compute the phases of the secondary jaws shall therefore be equally distributed around the azimuth with $\left(\phi_{i}=\phi_{0}+2(i-1) \pi / m_{s}, i \in\left[1, m_{s}\right]\right)$. Varying

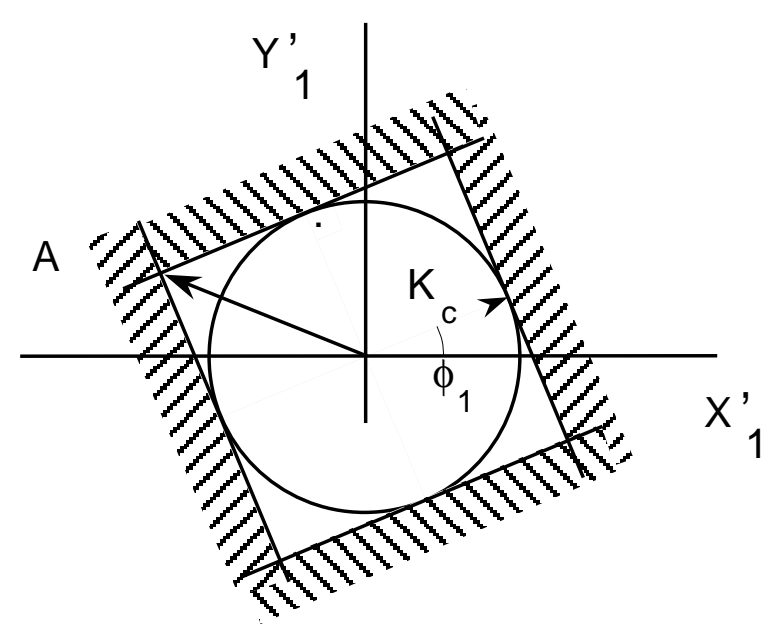

FIG. 2. The polygon delimited by the secondary jaws in the $X^{\prime}-Y^{\prime}$ plane. Here $m_{s}=4$. The scattering azimuth are chosen equidistant to form a square which minimizes the surface and the extension of the polygon. The largest angle passing the secondary jaws is $K_{4, \max }=\|O A\|=\sqrt{2} K_{c}$. 
$\phi_{0}$ rotates the polygon but does not modify the distribution of the combined secondary amplitude. The angle $\phi_{0}$ can therefore be freely chosen as long as isotropic scattering is considered. But in practice the outscattering rate is largest at $\phi=\alpha+\pi$, a value to which one jaw must be adjusted by choosing $\phi_{0}$ adequately.

\section{A finite number of collimators}

In a real collimation system, both the number $m_{p}$ of primary and $m_{s}$ of secondary jaws must be finite and small. The choice of $m_{p}$ and $m_{s}$ is made a bit complicated by an effective correlation between them. We first discuss the case of the primary jaws.

\section{Primary collimators}

We considered circular collimators in Sec. III to simplify our calculations. In practice, it is often desirable to define a circular primary aperture. One reason is to fit to a circular vacuum chamber which defines an approximately circular normalized aperture when integrated over an arc cell. Another reason might be the need to fit to a nearly circular dynamic aperture. On the other hand, in practice, the circular aperture must be approximated by flat jaws which have an adjustable distance to the beam. They shall be arranged to form a regular polygon to limit, at best, the primary amplitudes which are larger than the specified value $n_{1}$ (see Fig. 3). The phase advances of the secondary jaws shall be computed for the central impact points of the primary jaws (see Fig. 3), defined by the central azimuths $\left(\alpha_{i}=(i-1) \pi /\left[2\left(m_{p}-1\right)\right]\right.$, $\left.i \in\left[1, m_{p}\right]\right)$. At the central location, the primary aperture is $A_{0}=n_{1}$, while at the apex of the polygon it is

$$
A_{0, \max }=\frac{n_{1}}{\cos \left[\pi / 4\left(m_{p}-1\right)\right]},
$$

as deduced by trigonometry from Fig. 3. Primary impact maps are, of course, not limited to the central point of the jaw but rather continuously distributed all along the surface of the jaws. For later use, we define an approximate average primary amplitude over the whole azimuth $\alpha$ with

$$
\begin{aligned}
A_{0, \mathrm{eff}}\left(m_{p}\right) & =n_{\mathrm{eff}}\left(m_{p}\right) \\
& =\frac{1}{2}\left\{\left(n_{1}+\frac{n_{1}}{\cos \left[\pi / 4\left(m_{p}-1\right)\right]}\right)\right\} .
\end{aligned}
$$

With only $m_{p}=2$ primary jaws (usually oriented horizontally and vertically), the largest primary amplitude before scattering is $A_{0, \max }=\sqrt{2} n_{1} \simeq 1.41 n_{1}$ (see Fig. 3), which is a too large value if high performance is mandatory. With $m_{p}=3$ jaws, thus defining an octagonal primary aperture, a much better performance is obtained with $A_{0, \max }=n_{1} / \cos (\pi / 8) \simeq 1.08 n_{1}$ (see Fig. 3).

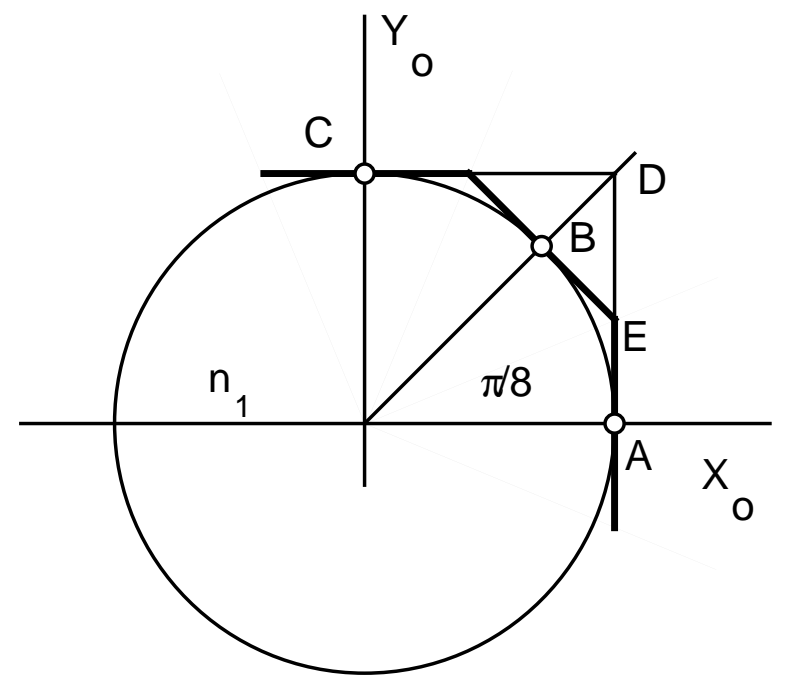

FIG. 3. The $X-Y$ primary impact distribution of the simulation on the primary collimator jaws. The small circles are the scattering sources of the central impact approximation for which the betatronic phase advances were computed (see text). With $m_{p}=2$, the largest amplitude before scattering is already $A_{0, \max }=\|O D\|=\sqrt{2} n_{1}$. With $m_{p}=3$, the jaws are arranged to form an octagonal primary aperture $A_{0, \max }=$ $\|O E\|=n_{1} / \cos (\pi / 8)=2 n_{1} /(2+\sqrt{2})^{1 / 2}$.

\section{Secondary collimators}

To help in choosing the number of secondary jaws, we give for a set of $m_{s}$ values in Table I the variable $K_{\max }\left(m_{s}\right)$ discussed in Sec. III B, the associated maximum secondary amplitude $A_{2, \max }\left(m_{s}\right)$, and the relative surface $S / K_{c}^{2}$ of the regular polygon which delimits the secondary halo in the phase space. Numerical values are computed with $n_{1}=6$ and $n_{2}=7$. Any number $m_{s}$ of secondary jaws can be considered, but above $m_{s}=4$ the changes per $m_{s}$ unit are small. With $A_{2, \max }\left(m_{s}=3\right)=9.4$, the case $m_{s}=3$ can be readily discarded and we further limit our discussion to $\left(m_{p}=3, m_{s}=4\right)$ and $\left(m_{p}=3, m_{s}=8\right)$. We must now take into account the effective primary amplitudes which limit the performance obtained by the secondary jaws. We therefore define an effective average of the maximum secondary amplitude

$$
A_{\mathrm{eff}, \max }\left(m_{p}, m_{s}\right)=\sqrt{n_{\mathrm{eff}}\left(m_{p}\right)^{2}+\frac{1}{4}\left[K_{c}+K_{\max }\left(m_{s}\right)\right]^{2}},
$$

with $n_{\text {eff }}\left(m_{p}\right)$ taken from Eq. (22). We also define the total number of jaws

$$
m_{\text {jaws }}=m_{p}\left(1+m_{s}\right) .
$$

Both $A_{\text {eff,max }}$ and $m_{\text {jaws }}$ are given in Table II.

The difference of the effective performance $\delta A_{\max }=$ 0.3 between the two cases $\left(m_{p}=3, m_{s}=4\right)$ and $\left(m_{p}=\right.$ $3, m_{s}=8$ ) (see Table II) is marginal, ruling out the case $\left(m_{p}=3, m_{s}=8\right)$. To make full use of eight secondary jaws, five primary jaws must be considered, with a result 
TABLE I. Extension of the secondary halo for different numbers $m_{s}$ of pairs of secondary jaws per primary impact point. The variable $m_{s}$ is also the order of the polygon discussed in Sec. IIIB. The expressions for $K_{\max }$ are obtained by the geometry of regular polygons and the maximum amplitudes are given by $A_{\max }^{2}=n_{1}^{2}+K_{\max }^{2}$. We used the collimator apertures $n_{1}=6$ and

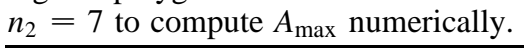

\begin{tabular}{cccccc}
\hline \hline$m_{s}$ & $K_{\max }$ & $A_{\max }^{2}$ & $A_{\max }$ & $S / K_{c}^{2}$ & $S / K_{c}^{2}$ \\
\hline 3 & $2 K_{c}$ & $4 n_{2}^{2}-3 n_{1}^{2}$ & 9.4 & $3 \sqrt{3}$ & 5.19 \\
4 & $\sqrt{2} K_{c}$ & $2 n_{2}^{2}-n_{1}^{2}$ & 7.9 & 4 & 4.00 \\
8 & $2 K_{c} /(2+\sqrt{2})^{1 / 2}$ & $\left(4 n_{2}^{2}-2 n_{1}^{2}+\sqrt{2} n_{1}^{2}\right) /(2+\sqrt{2})$ & 7.2 & $8 \sqrt{(2-\sqrt{2}) /(2+\sqrt{2})}$ & 3.31 \\
$\infty$ & $K_{c}$ & $n_{2}$ & 7 & $\pi$ & 3.14 \\
\hline \hline
\end{tabular}

close to the ultimate limit $A_{\max }=n_{2}$, but at the price of a quite prohibitive number of jaws amounting to 45 (see Table II).

We therefore further consider the case $m_{p}=3$ with $m_{p}=4$ secondary collimators per primary one. The phases in Table III are computed with Eqs. (12) for the central impacts on the primary jaws $\alpha=0, \pi / 4, \pi / 2$ with four equidistant scattering angles $\phi_{i}=[\alpha+(i-$ 1) $\pi / 2, i=1,4]$. These correlated phases constitute a specification for an optic to offer the smallest secondary halo extension for the given number of jaws.

\section{Simulation for continuous primary impact}

To check the relevance of the effective maximum amplitude of Eq. (23), we integrated numerically the amplitude distribution of the secondary halo with a simple simulation program. We used the primary and secondary apertures $n_{1}=6$ and $n_{2}=7$. Primary impacts are uniform along the inner surface of the jaws. Scattering angles are uniform in the $K-\phi$ plane. The tracking is made with the transfer matrix (3) in which the phases $\left(\mu_{y}, \mu_{x}\right)$ are taken from Table III. At each collimator it is verified if the particle touches a jaw. The particles surviving all secondary collimators are added to a $A_{x}-A_{y}$ plot, thus building the density distribution $d^{2} N / d A_{x} d A_{y}$ of the secondary halo (see Fig. 4), and added also to a combined amplitude distribution $d N / d A$ (see Fig. 5). The case $m_{s}=8$ is also explored and added to Fig. 5 .

TABLE II. Effective maximum amplitude of the secondary halo $A_{\text {max,eff }}$ and total number of jaws $m_{\text {jaws }}$ as a function of the number of primary and secondary jaws $m_{p}$ and $m_{s}$. The primary jaws are arranged to form a regular polygon in the normalized plane $\left(X_{1}, Y_{1}\right)$. The secondary jaws are arranged to contain the scattering angles inside another regular polygon located in the normalized plane $\left(X_{1}^{\prime}, Y_{1}^{\prime}\right)$. The betatronic phase advances between primary and secondary jaws are the optimum ones; see text.

\begin{tabular}{lccc}
\hline \hline$m_{p}$ & $m_{s}$ & $A_{\text {max,eff }}$ & $m_{\text {jaws }}$ \\
\hline 3 & 4 & 7.6 & 15 \\
3 & 8 & 7.3 & 27 \\
5 & 4 & 7.5 & 25 \\
5 & 8 & 7.1 & 45 \\
\hline \hline
\end{tabular}

The effective maximum amplitude of Eq. (23) fits well to the end of the distribution $d N / d A$ and is therefore a good indicator of the limit of the secondary halo. The distributions shown in Fig. 5 confirm that the case $\left(m_{s}=3, m_{p}=8\right)$ is not worth the additional hardware investment while four secondary collimators for each of the primary azimuths, i.e., twelve pairs of secondary jaws with three primary collimators, is a quite good choice. This conclusion about the number of jaws was already obtained by Kaltchev [3,6] who developed a numerical algorithm to minimize the size of a polygon in the $X^{\prime}-Y^{\prime}$ plane.

\section{E. Existing solutions}

With a symmetric optic $\left[\mu_{x}(s)=\mu_{y}(s)\right]$, the secondary halo is cut at $A_{\mathrm{sec}}=1.32 n_{2}$ [2] with a ratio

TABLE III. Secondary collimator locations $\mu_{x}$ and $\mu_{y}$ and jaw orientations $\alpha_{J}$ for three scattering azimuths $\alpha$ and four scattering angles $\phi$. One can add $\pi$ to any of these phases but then $\alpha_{\text {jaw }}$ must be reevaluated. It is assumed that jaws are mounted in transversely opposite pairs, i.e., for each entry in the table there is a jaw at $\alpha_{J}$ and one at $\alpha_{J}+\pi$, for operational reasons explained in Sec. III A. We listed the value $\alpha_{J}$ which is closer to the first quadrant. The lines of the table where $\phi=\alpha$ or $\phi=\alpha+\pi$ correspond to plane scattering and define a $1 \mathrm{D}$ collimation system. The existing collimation systems in proton colliders cut on plane scattering and only with horizontal and vertical primary jaws, for example, have primary and secondary jaws corresponding to lines $1,2,9$, and 10 of the table.

\begin{tabular}{ccccc}
\hline \hline$\alpha$ & $\phi$ & $\mu_{x}$ & $\mu_{y}$ & $\alpha_{J}$ \\
\hline 0 & 0 & $\mu_{0}$ & - & 0 \\
0 & $\pi$ & $\pi-\mu_{0}$ & - & 0 \\
0 & $\pi / 2$ & $\pi$ & $3 \pi / 2$ & $\mu_{0}$ \\
0 & $-\pi / 2$ & $\pi$ & $3 \pi / 2$ & $-\mu_{0}$ \\
$\pi / 4$ & $\pi / 4$ & $\mu_{0}$ & $\mu_{0}$ & $\pi / 4$ \\
$\pi / 4$ & $5 \pi / 4$ & $\pi-\mu_{0}$ & $\pi-\mu_{0}$ & $\pi / 4$ \\
$\pi / 4$ & $3 \pi / 4$ & $\pi-\mu_{0}$ & $\pi+\mu_{0}$ & $\pi / 4$ \\
$\pi / 4$ & $-\pi / 4$ & $\pi+\mu_{0}$ & $\pi-\mu_{0}$ & $\pi / 4$ \\
$\pi / 2$ & $\pi / 2$ & - & $\mu_{0}$ & $\pi / 2$ \\
$\pi / 2$ & $-\pi / 2$ & - & $\pi-\mu_{0}$ & $\pi / 2$ \\
$\pi / 2$ & $\pi$ & $\pi / 2$ & $\pi$ & $\pi / 2-\mu_{0}$ \\
$\pi / 2$ & 0 & $\pi / 2$ & $\pi$ & $\pi / 2+\mu_{0}$ \\
\hline \hline
\end{tabular}




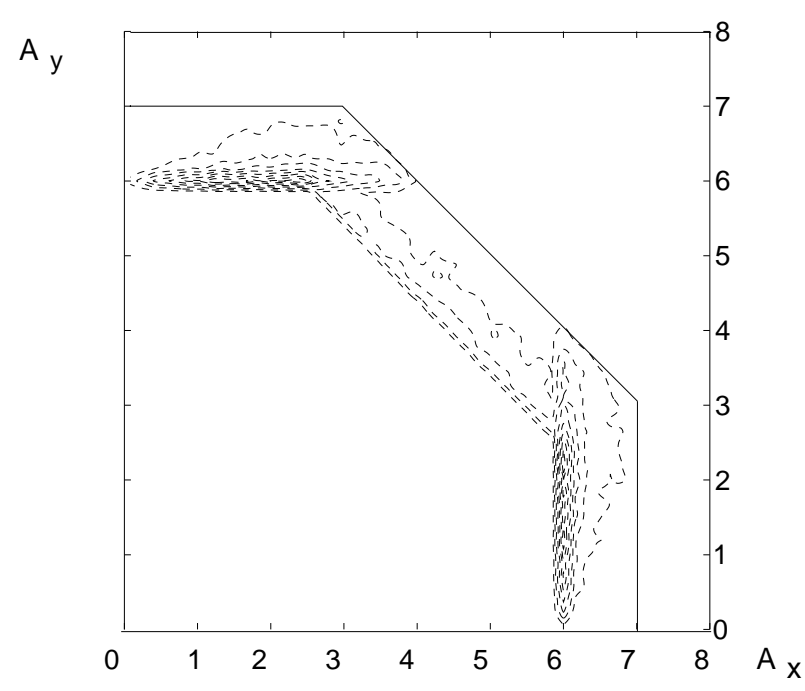

FIG. 4. The contour plot of the distribution $d^{2} N / d A_{x} d A_{y}$ of the secondary halo in the case $m_{p}=3$ and $m_{s}=4$ with continuous primary impact distribution. This distribution is obtained with isotropic scattering. The normalized amplitudes $A_{x}$ and $A_{y}$ are in rms beam units. We used collimator apertures $n_{1}=6$ and $n_{2}=7$. The two octagons of inner radii $n_{1}$ and $n_{2}$ indicate that the secondary halo is almost entirely contained inside these limits.

$n_{2} / n_{1}=7 / 6$. The present best performance obtained with a modulated optic for the LHC collimation insertion is $A_{\mathrm{sec}}=1.21 n_{2}$ [3]. It was emphasized in former studies $[2,3]$ that cutting efficiently on large amplitudes

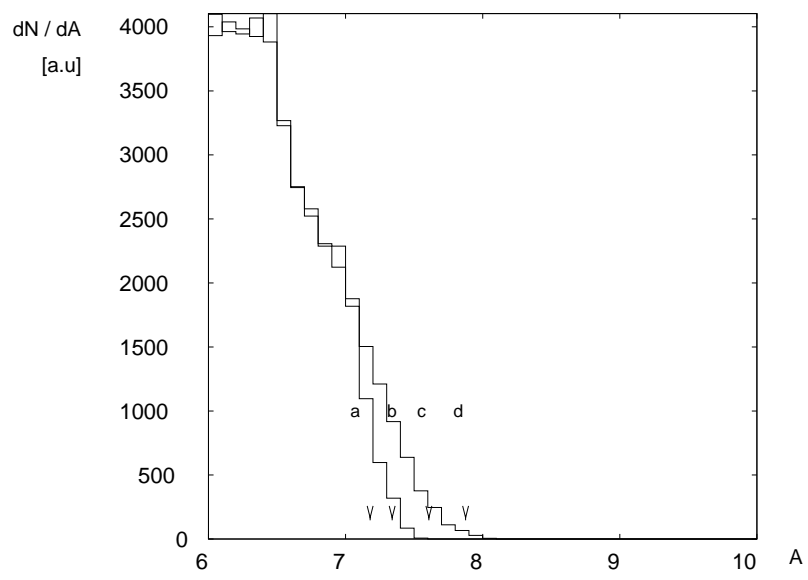

FIG. 5. The distribution $d N / d A$ for $m_{p}=3$ and $m_{s}=4$ (upper curve) compared to $m_{p}=3$ and $m_{s}=8$ (lower curve). We used collimator apertures $n_{1}=6$ and $n_{2}=7$. The combined normalized amplitude $A$ is in normalized rms beam units. This distribution is obtained with isotropic scattering. The arrows $a$ and $d$ correspond to $A_{4, \max }$ and $A_{8, \max }$ computed with central impacts and are taken from Table II. The arrows $b$ and $c$ are the effective limits of Eq. (23) for the same two cases. The latter ones are much better estimators of the upper limits if the fading ends of the spectra are neglected. associated to orthogonal scattering $(\phi=\alpha \pm \pi / 2)$ requires large phase modulation, i.e., large $\mu_{y}-\mu_{x}$ values, along the cleaning insertion. This argument was right but incomplete. Strict correlation of the phase advances $\mu_{x}$ and $\mu_{y}$ is mandatory, and the maximum modulation $\mu_{y}-\mu_{x}=\pi / 2$ is needed for some jaws (see Table III). While it may be unfair to compare the performance of existing optics to our nearly ultimate limit $A_{\text {max,eff }}=1.08 n_{2}$ obtained with a yet virtual one, a potential gain remains to be exploited with an optic which satisfies the phase advances specified in Table III.

\section{F. Sensitivity to errors}

Phase error. The sensitivity of the cut of the secondary amplitude associated to a phase error $\nu_{x, y}$ between a primary and a secondary jaw is obtained by computing first the modified spatial extensions $\tilde{X}_{2}$ and $\tilde{Y}_{2}$ of a particle of amplitude $A_{2}=n_{2}$ at the new location of the jaw. With $K=K_{c}$ in Eq. (8) for $X_{2}$,

$\tilde{X}_{2}=n_{1} \cos \alpha \cos \left(\mu_{x}+\nu_{x}\right)+K_{c} \cos \phi \sin \left(\mu_{x}+\nu_{x}\right)$.

By multiplying the terms on the right-hand side by the identity $\cos \alpha_{J} / \cos \alpha_{J}$ and using Eqs. (14) and (12), we get

$$
\tilde{X}_{2}=n_{2} \cos \alpha_{J} \cos \nu_{x},
$$

and, similarly, $\tilde{Y}_{2}=n_{2} \sin \alpha_{J} \cos \nu_{y}$. Considering a maximum phase error $\nu_{x, y}<\nu$, the spatial extension of the particle of amplitude $n_{2}$ is therefore smaller than $n_{2}$ by $\cos \nu$. The jaw is therefore intercepting the particles with an amplitude $\tilde{A}_{2}>n_{2} / \cos \nu$. At first order in $\nu$, we get $\delta A / A=(\tilde{A}-A) / A \approx \nu^{2} / 2$. Numerically, using a phase error in the collimation insertion of $\nu<5^{\circ}$, the relative error on the amplitude cut is at most $\delta A / A<5 \times 10^{-3}$. The phases $\mu_{x, y}$ are the sole variables governing the amplitude cut in the case of a linear and uncoupled insertion, taking apart the trivial transverse mispositioning of a collimator, which must be adjusted during the operation of the ring.

Linear coupling. In a proton collider, the linear coupling must be corrected down to a level where the period of coupling is much larger than the betatron period [7]. The relative variation of the amplitudes shall thus be small, or $\delta A_{x, y} / A \ll 1$ across the collimation insertion. On the other hand, the scattering process which populates the secondary halo is a strong source of local stochastic coupling, for the angular distribution $d N / d \phi$ being almost isotropic. This effect is intrinsically taken into account in our approach and the small effect of the residual linear coupling can be safely neglected. 
Nonlinear effects. We will use a very simplified approach of nonlinear perturbations of the regular betatron motion. We first make the reasonable assumption that the long-term dynamic aperture $A_{\mathrm{dyn}}$ is larger than the secondary aperture at which the secondary halo is approximately cut, say $A_{\mathrm{dyn}}>n_{2}+1$. With this assumption, the motion is nearly regular in the range of amplitudes considered in our problem [8]. The motion can be crudely described by the superimposition of a coupling effect, i.e., a slow exchange of $A_{x}$ and $A_{y}$, and of a slow variation of the combined amplitude $A$, often called amplitude smearing. The amplitude $A$ of every particle drifts slowly between two limits $A_{\min }<A<A_{\max }$, with $A_{\min }$ and $A_{\max }$ being correlated by a monotonous function. Coupling and smearing can both be neglected when considering a single pass through the collimation insertion, similarly to the case of the residual linear coupling. The particles which survive to their first pass across the collimation insertion, and which thus have an amplitude $n_{1}<A<n_{2}+1$, will do a few turns before being absorbed. The net efficiency of the system will be only slightly degraded by the small change of amplitude associated to the smearing during these few turns, while the coupling will just modify the azimuth of the second impact, which has little or no effect on the efficiency of the system.

Primary aperture and nonlinear effects. An additional effect, which is not related to collimation strictly speaking, is, nevertheless, worth being briefly discussed. A proton which is just to touch a primary collimator is also subject to amplitude smearing and oscillates in a range $\tilde{n}_{1}<A<n_{1}$. Therefore the effective primary aperture is $\tilde{n}_{1}$ instead of $n_{1}$. The smearing function must therefore be evaluated in addition to $A_{\mathrm{dyn}}$ when designing a collider.

\section{G. Isotropic and real scattering}

If the use of isotropic scattering is adequate to compare different jaw arrangements, real scattering must be considered to quantify the performance of a system in absolute terms. We give here only a brief outlook of a discussion made in [1] where a complete algorithm is described which combines real scattering and multiturn tracking. In first approximation, elastic scattering of protons in matter is dominated by multiple Coulomb scattering. The angular distribution after scattering of a proton of momentum $p$ through one interaction length of matter is Gaussian with a rms width $\sigma_{\mathrm{mcs}}^{\prime}=$ $a p^{-1} \simeq 3 \times 10^{-5} p^{-1}[\mathrm{rad}, \mathrm{TeV} / c]$, considering here an aluminum jaw. The quantity $\sigma_{\mathrm{mcs}}^{\prime}$ is compared to the rms beam divergence $\sigma_{\beta}^{\prime}=\left(\epsilon_{n} m_{p} / \beta p\right)^{1 / 2}=b p^{-1 / 2} \simeq$ $6 \times 10^{-6} p^{-1 / 2} \mathrm{rad}$ with the proton mass $m_{p}=0.94 \times$ $10^{-3} \mathrm{TeV} / c^{2}$ and an average betatronic wavelength $\beta \simeq$ $100 \mathrm{~m}$. In a proton collider, we use a normalized emittance $\epsilon_{n} \simeq 4 \times 10^{-6} \mathrm{~m}$. The isotropic scattering model is adequate if the real scattering distribution is wider than the angular cut $K_{c}$ made by the collimators, i.e., if

$$
\sigma_{\mathrm{mcs}}^{\prime} \geq K_{c} \sigma_{\beta}^{\prime},
$$

with $K_{c}$ taken from Eq. (10). With $n_{1}=6$ and $n_{2}=$ 7 , the crossover momentum $p=\left(a / K_{c} b\right)^{2} \simeq 2 \mathrm{TeV} / c$ deduced from the equality in Eq. (27) defines the limit below which the condition (27) is satisfied. Above this momentum, the isotropic model substantially overestimates the size of the secondary halo cut by the collimators. At the injection momentum of LHC, or $p=0.45 \mathrm{TeV} / c$, the performance of the cleaning insertion is $A_{\text {isotropic,cut }}=$ 8.4 [3]. The limit obtained with a numerical model which includes real scattering, tertiary halo, and multiturn tracking is $A_{\text {cut }}=8.0$ [1], a slightly better value than the result obtained with the isotropic model. This indicates that the range of the crossover momentum is quite large.

\section{H. Secondary halo and quench levels}

The link between the edge of the secondary halo and the quench levels in superconducting magnets is not direct. It is discussed in Ref. [1] and briefly outlined here. An aperture limitation in the ring delimits a small volume in phase space, in which protons will be captured locally. The integral of the flux of the halo in that small volume must be compared to the quench limit. If the edge $A_{\text {cut }}$ of the distribution $d N / d A$ of the secondary halo is smaller than the aperture of the ring $A_{\text {ring }}$, the secondary halo induces no direct losses. Tertiary losses, made of protons elastically scattered off the secondary collimators, must then be considered and compared to the quench limit. On the other hand, it is always important to satisfy the condition $A_{\text {cut }}<A_{\text {ring }}$ because of the steep slope of $d N / d A$ below $A_{\text {cut }}$ (see Fig. 5).

\section{MOMENTUM COLLIMATION}

We restrict our discussion to a momentum cleaning insertion installed in a straight section, where the dispersion function is a betatronic trajectory. In that case, the condition $D^{\prime} / D=-\alpha_{x} / \beta_{x}$, or, equivalently, $\chi^{\prime}=0$ [see Eqs. (1) and (2)], must be satisfied at the primary collimator [2,9] to ensure that the cut made on the secondary halo does not depend on the relative momentum offset $\delta_{p}$. It also strictly reduces the treatment of the momentum collimation to the betatronic case in a straight section [2], while outside the straight section the transverse offset $x_{\beta}$ and $x_{\delta_{p}}=D \delta_{p}$ must, of course, be distinguished.

In the usual case of a ring without substantial vertical dispersion and in contrast with the betatron halo which may drift away from the beam in all transverse directions, momentum losses are concentrated in the horizontal plane. The most demanding case occurs at ramping when off-bucket protons are lost. Most of these protons keep their initial betatronic amplitude at injection [10] and are therefore confined in the range of betatronic amplitudes $A_{x, y} \approx 2$. It is therefore enough to use 
a single horizontal primary collimator, to which four secondary collimators must be associated, following the conclusions of Sec. IIIC. Their locations correspond to the case $\alpha=0$ of Table III, and they limit the components of the betatron vector after scattering to $\vec{A}_{1}=$ $\left(n_{1}, K_{c}, \approx 2, K_{c}\right)$.

In the arc of a ring, the aperture limitation for a particle with momentum offset is located near horizontally focusing quadrupoles where both $\beta_{x}$ and $D_{x}$ are at their maximum. In addition, with $\beta_{y} \ll \beta_{x}$ it is thus adequate to fit the largest horizontal secondary excursion $A_{x, \beta}+$ $D \delta_{p}$ of the secondary halo with the aperture $N_{\text {arc }}=N_{x \text {,arc }}$ at that location. The straight sections of a ring need not be considered for momentum collimation since the dispersion is usually suppressed in these areas.

\section{A. Amplitude cut with momentum offset}

In the general case, a particle reaches the primary collimator with a mixing of betatron amplitude and momentum offset. With the dispersion $\chi_{1}$ at the primary collimator, and using the approximation of slow diffusion (see Sec. III), we write

$$
n_{1}=\chi_{1} \delta_{p}+X_{\beta}=\chi_{1} \delta_{p}+A_{x, \beta}
$$

and define the largest momentum offset which can pass the primary collimator as $\delta_{c}=n_{1} / \chi_{1}$ with $A_{x, \beta}=0$. After scattering and the cut of the amplitude by the secondary collimators, the maximum horizontal betatronic amplitude is $A_{x, \beta}=\left[\left(n_{1}-\chi_{1} \delta_{p}\right)^{2}+K_{c}^{2}\right]^{1 / 2}$. Expanding $A_{x, \beta}$ with Eq. (10), the maximum horizontal excursion in the arc is

$$
\begin{aligned}
& X_{\max }\left(n_{1}, \chi_{1}, \delta_{p}\right) \\
& \quad=\chi_{\operatorname{arc}} \delta_{p}+\left(\chi_{1}^{2} \delta_{p}^{2}-2 n_{1} \chi_{1} \delta_{p}+n_{2}^{2}\right)^{1 / 2}
\end{aligned}
$$

and is plotted in Fig. 6. The largest allowed excursion $X_{\max }\left(n_{1}, \chi_{1}, \delta_{c}\right)=N_{\text {arc }}$ fixes

$$
\delta_{c}\left(n_{1}\right)=n_{1} / \chi_{1}=\left[N_{\mathrm{arc}}-\left(n_{2}^{2}-n_{1}^{2}\right)^{1 / 2}\right] \chi_{\mathrm{arc}},
$$

obtained with $\delta_{p}=\delta_{c}\left(n_{1}\right)$ in Eq. (29). Should large $n_{1}$ values be considered (see Fig. 6), the large $X_{\max }$ excursion at small $\delta$ values would be cut at the betatron cleaning insertion. The system is completely fixed by choosing $n_{1}$ and computing the dispersion which is needed at the primary collimator,

$$
\chi_{1}\left(n_{1}\right)=\frac{n_{1}}{\delta_{c}}=\frac{n_{1} \chi_{\mathrm{arc}}}{N_{\mathrm{arc}}-\left(n_{2}^{2}-n_{1}^{2}\right)^{1 / 2}} .
$$

As for the choice of $n_{1}$, a lower limit $n_{1, \min }$ is fixed by the acceptable effective cut of the primary horizontal betatronic amplitude at the edge of the bucket $n_{\text {edge }}=$ $n_{1}\left(1-\delta_{b} / \delta_{c}\right)$ with $\delta_{b}$ the bucket width. In practice,

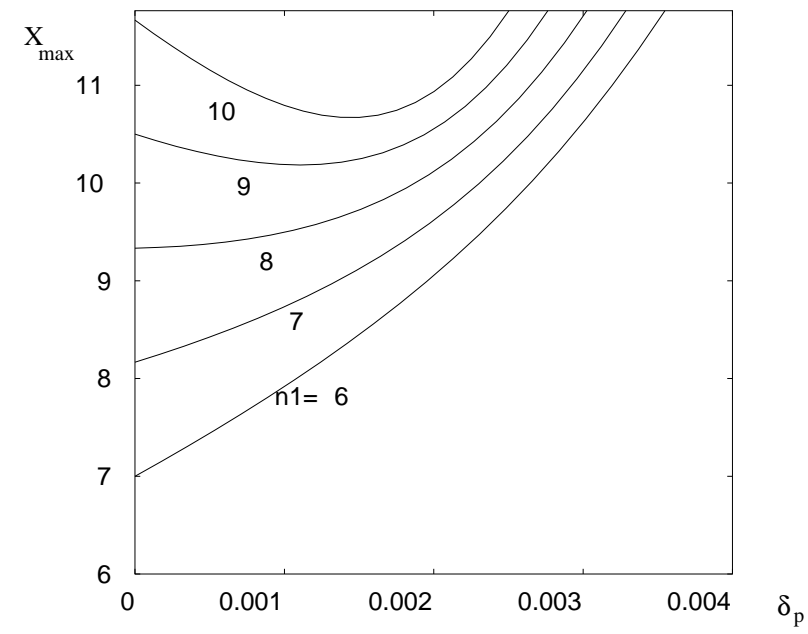

FIG. 6. The maximum transverse normalized excursion $X_{\max }$ of a particle as a function of the relative momentum offset $\delta_{p}$ and of the primary collimator aperture $n_{1}$. Each curve is ended at $\delta_{p}=\delta_{c}\left(n_{1}\right)$ where $X_{\max }=N_{\text {arc }}=11.8$, a case study for LHC for which we fixed the ratio $n_{2} / n_{1}=7 / 6$.

a high enough $\chi_{1}$ must be obtained by matching the optic such that the corresponding $n_{1}$ value obtained with Eq. (31) is larger than $n_{1, \min }$.

\section{SUMMARY}

We derived the correlated betatronic phase advances between primary and secondary jaws which allow one to cut the amplitude of the secondary halo of a two-stage collimation system down to the aperture of the secondary collimators. We showed that an infinite number of jaws would be necessary to reach that limit. We derived a precise estimator of the effective extension of the secondary halo for a finite number of jaws. We give a specification that an optic must satisfy for the case of a collimation system made of three primary jaws and twelve secondary ones. We also specified the properties of a momentum cleaning insertion.

\section{ACKNOWLEDGMENTS}

The author is indebted to T. Trenkler who pointed him to Eq. (20) and to part of the subsequent deductions, and to D. Kaltchev with whom he had numerous useful discussions.

[1] N. Catalan Lasheras, G. Ferioli, J. B. Jeanneret, R. Jung, D. I. Kaltchev and T. Trenkler, in Proceedings of the International Symposium on Near Beam Physics, edited by D. Carrigan and N. Mokhov (Fermilab, Batavia, IL, 1997); CERN LHC Report No. 156, 1998.

[2] T. Trenkler and J.B. Jeanneret, Part. Accel. 50, 287 (1995). 
[3] D. I. Kaltchev, M. K. Craddock, R. V. Servranck, and J. B. Jeanneret, in Proceedings of the Particle Accelerator Conference, Vancouver, Canada, 1997, edited by M. K. Craddock et al. (IEEE, Piscataway, NJ, 1998), p. 1432; CERN LHC Project Report No. 134, 1997.

[4] M. Seidel, Desy Report No. 94-103, Hamburg, Germany, 1994.

[5] N. Catalan Lasheras, G. Ferioli, and J. B. Jeanneret, in Proceedings of the Sixth European Particle Accelerator Conference, Stockholm, 1998, edited by S. Myers et al. (Institute of Physics, Philadelphia, PA, 1998), p. 242; CERN LHC Project Report No. 185, 1998.
[6] D. I. Kaltchev, M. K. Craddock, R. V. Servranck, and J. B. Jeanneret, in Proceedings of the Fifth European Particle Accelerator Conference, Sitges, Spain, 1996, edited by S. Myers et al. (Institute of Physics, Bristol, England, 1996), p. 1432; CERN LHC Project Report No. 37, 1996.

[7] H. Wiedemann, Particle Accelerator Physics (SpringerVerlag, Berlin, 1995), Vol. 2, p. 29.

[8] F. Schmidt, DESY HERA Report No. 88-02, 1988.

[9] P. J. Bryant and E. Klein, CERN SL Report No. 92-40, 1992.

[10] N. Catalan Lasheras, CERN LHC Project Report No. 200, 1998. 